\title{
The Pitfalls of Febrile Jaundice. A Case Report
}

\author{
Maria Obreja ${ }^{1 *}$, Andra Teodor ${ }^{1,2}$, Daniela Leca ${ }^{1,2}$, Alexandr Ceasovschih ${ }^{3}$, Egidia Miftode ${ }^{1,2}$ \\ 1 "Sfânta Parascheva" Infectious Diseases Hospital lași, România \\ 2 "Grigore T. Popa" University of Medicine and Pharmacy, lași,România \\ ${ }^{3}$ Department of Medicine, "Sfântul Spiridon" Emergency Clinical Hospital, Iași, România
}

\begin{abstract}
Jaundice in sepsis is usually caused by cholestasis, and its onset can precede other manifestations of the infection. Inflammation-induced cholestasis is a common complication in patients with an extrahepatic infection or those with inflammatory processes. We describe the case of a 47 years old female who presented with low back pain and paravertebral muscular contracture. She subsequently developed a cholestatic syndrome with clinical manifestations such as jaundice, followed by fever and sepsis with multiple organ dysfunction. Initially labeled as biliary sepsis, the diagnosis was crucially reoriented as the blood cultures were positive for Streptococcus pyogenes and the magnetic resonance imaging (MRI) findings suggested spondylodiscitis as well as a paravertebral abscess.
\end{abstract}

Keywords: lower back pain, jaundice, fever, blood cultures, S.pyogenes, sepsis

Received: 02 February 2016 / Accepted: 03 April 2016

\section{INTRODUCTION}

A great variety of bacterial infections frequently associates jaundice and hepatic dysfunction. Jaundice can result either directly from bacterial products or as a consequence of the host's reaction to the infection. Although jaundice can be an isolated sign, it is usually associated with cholestasis. In the critically ill patient, jaundice or the associated cholestasis complicates the clinical picture, causing a difficult diagnosis and management.

Jaundice in sepsis can be present a few days before bacterial discharge, other clinical signs and even before an infection becomes apparent [1].

\section{CASe Presentation}

We present the case of a 47 years old woman who came to the Emergency Room complaining of a painful low back pain syndrome, bilateral sciatic pain, lumbar paravertebral muscular contraction and difficulty in walking. She was admitted to the Neurosurgery Emergency Hospital. The clinical examination showed a conscious and cooperating patient, with normal coloured skin, plantar clavus, and onychomycosis of the feet. She had a normal pulmonary auscultation; blood pressure was 120/70 $\mathrm{mmHg}$ and heart rate 80 beats/minute, no abdominal pain on palpation, normal bowel sounds, liver size was within normal limits and no frequency or urgency of micturition, dysuria or haematuria. An MRI revealed L5-S1 right paramedian disc protrusion with right S1 compression and L5 intraforaminal bilateral disc fingerprint. During her five day stay in the Neurosurgical Clinic, her general status got worst, and the patient developed intense jaundice, asthenic syndrome, and fever. The biologic tests showed an important inflammatory syndrome $\left(\mathrm{WBC}=18.2 \times 10^{3} / \mu \mathrm{l}\right.$ with $92,4 \%$ neutrophiles, ESR $=50 \mathrm{~mm} / \mathrm{hr}$ ) and cholestasis (Total bilirubin $=7,56 \mathrm{mg} / \mathrm{dl}$, Indirect bilirubin $=0,65 \mathrm{mg} /$ $\mathrm{dl}$, Direct bilirubin=6,91 $\mathrm{mg} / \mathrm{dl}$ ), mild anemia (with RBC: $3.66 \times 106 / \mu \mathrm{l}, \mathrm{Hb}=11 \mathrm{~g} / \mathrm{dl}, \mathrm{Ht}=33 \%$ ), severe thrombocytopenia (PLT: $\left.9 \times 10^{3} / \mu \mathrm{l}\right), \mathrm{ALP}=427 \mathrm{IU} / \mathrm{l}$, ALAT $=77 \mathrm{IU} / 1, \mathrm{ASAT}=53 \mathrm{IU} / \mathrm{l}$. This clinical picture raised the suspicion of an acute cholecystitis where the colicative pain was irradiating in the lumbar region. The thorax and abdominal CT scan showed a $25 \mathrm{~mm}$ calculus inside the gallbladder. Considering the persistent febrile syndrome and the imaging tests results which temporary excluded a surgical, neurosurgical or gastrointestinal pathology, a sepsis of unknown origin

\footnotetext{
Correspondence to: Maria Obreja, Address: Str. Sarmisegetuza, Nr. 5, lași, România. E-mail: mmariaobreja@gmail.com Andra Teodor: Universitatea de Medicina si Farmacie "Gr.T.Popa" lasi, Str. Universitatii nr.16 700115 lasi, Romania

Daniela Leca: Universitatea de Medicina si Farmacie "Gr.T.Popa" lasi, Str. Universitatii nr.16 700115 lasi, Romania

Alexandr Ceasovschih: "Sfantul Spiridon" Emergency Clinical Hospital, lasi, Romania. Adresa: Bd. Independentei nr. 1, cod 700111, lasi, Romania

Egidia Miftode: Universitatea de Medicina si Farmacie "Gr.T.Popa" lasi, Str. Universitatii nr.16 700115 lasi, Romania
} 
was suspected and the patient was sent to an Infectious Disease Clinic. Following haemocultures, antibiotic therapy was initiated with Vancomycin 2g, Ertapenem $1 \mathrm{~g}$, and Co-trimoxazole 480mg. During the hospital stay, the febrile syndrome went into remission, but the biological picture got worse with inflammatory and cholestatic tests augmented (Figure 1). Because the patient's general state continuously deteriorated, further surgical evaluation was considered necessary.

The patient was redirected to the Surgery Clinic of "Sfantul Spiridon" Hospital, Iasi, with suspected mechanical obstructive jaundice. However, magnetic resonance cholangio pancreatography (MRCP) failed to show any gallbladder stones inside the bile duct. After initiation of antialgic, antispastic, antibiotic (amoxiciline+clavulanic acid) and antisecretory therapy, bilirubin values started to fall, but the patient became febrile. Haemocultures were positive for Streptococcus pyogenes. The biological picture of persistent neutrophilic leukocytosis (WBC: $16.6 \times 10^{3} / \mu \mathrm{l}$ with 82,7 $\%$ neutrophiles) determined the patient's transfer to the Infectious Disease Clinic.

On presentation at the Infectious Disease Clinic, the patient showed severely influenced general status with hyperalgic lumbar syndrome, urine catheter, productive cough, shortness of breath. Paraclinical investigations revealed an important inflammatory syndrome, with the suggestive aspect of bronchopneumonia on the chest x-ray. Treatment with Ciprofloxacine 400mg and Ampiciline $10 \mathrm{~g}$ was initiated. Due to the persistent lumbar symptomatology, another MRI was obtained, which revealed multiple level spondilodiscitis and associated fused collections along the psoas muscles. The paravertebral posterior muscles had an infiltrative appearance, and bilateral abscesses were highlighted. The bacteriological tests also suggested a urinary infection with Klebsiella pneumoniae. Treatment with Ciprofloxacine was stopped, and Amikacine 1g was added. The evolution was slowly favorable during the 44 days

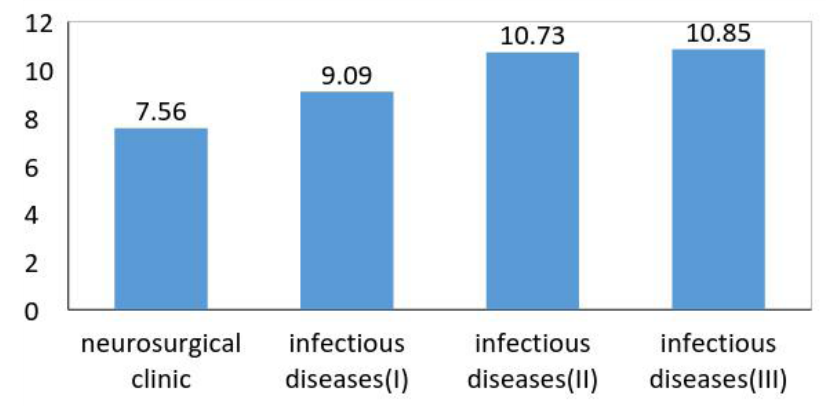

Fig. 1. Increase in total bilirubin levels of antibioterapy. The final laboratory tests showed a moderate inflammatory syndrome without leukocytosis $\left(\mathrm{CRP}=92 \mathrm{mg} / \mathrm{l}, \mathrm{ESR}=140 \mathrm{~mm} / \mathrm{hr}, \mathrm{WBC}=6,75 \times 10^{3} /\right.$ $\mu \mathrm{l}$ with $39,9 \%$ neutrophiles), persistent mild anemia ( $\mathrm{RBC}=2.55 \times 106 / \mu \mathrm{l}, \mathrm{Hb}=7,5 \mathrm{~g} / \mathrm{dl}, \mathrm{Ht}=22,7 \%$ ), moderate cytolysis hepatic syndrome $($ ALAT $=65 \mathrm{IU} / \mathrm{l}, \mathrm{ASAT}=38$ IU/l) with normal levels of bilirubin and reactive thrombocytosis caused by infection (PLT $=480 \times 10^{3} /$ $\mu \mathrm{l})$. At discharge, clinical exam revealed diminished pain and the patient resumed walking using a frame.

\section{DISCUSSION}

The liver plays a crucial role during the septic process. It contributes to the elimination of the infective agent. Sepsis also affects the liver through haemodynamic changes or by directly assaulting the hepatocytes, or by a combination of these two mechanisms. Liver dysfunction caused by sepsis is one of the severity SOFA score criteria [2].

It also represents one of the multiple organ dysfunction syndromes (MODS) components and is usually associated with a bad prognosis, though the incidence is still not well known. While the liver plays a major role in controlling some metabolic pathways, the homeostasis, and some defense mechanisms, liver dysfunction is seen as a consequence of shock and tissue hypoperfusion [3].

The results of an observational study on 283 critically ill patients supported the idea that sepsis is one of the most important promoters of hyperbilirubinemia. Other histopathological studies on patients with sepsis showed a predominantly intrahepatic cholestasis [3].

Cholestasis in sepsis can also be produced by other mechanisms such as biliary obstruction, cholangitis and ischemic liver injury during the septic shock. Generally, the hyperbilirubinemia is considered when the values of bilirubin are between 2 and $10 \mathrm{mg} / \mathrm{dl}$. It associates an elevated seric ALP value, but no more than 2 or 3 times the normal value. Most of the times, it is not associated with very high values of transaminases [4], in agreement with the findings in our case presentation.

Cholestatic jaundice caused by bacteremia may occur, in more than one-third of the patients, one to nine days before obtaining a positive haemoculture [5]. Because jaundice became evident before confirmation of a bacteriemia, a diagnosis is frequently orientated to a digestive aetiology. 
When there is no objective proof of biliary obstruction, the aetiology is probably a systemic infection. Sepsis is the most common cause of jaundice and cholestasis in intensive care units [1].

Jaundice is frequently associated with severe infections such us pneumonia, gram-negative bacterial sepsis or septic shock. Jaundice has been reported to be related to sepsis caused by gram-positive bacteria [6].

The clinical manifestations of Streptococcus pyogenes infection are among the most diverse: from a simple pharyngitis or skin infections to glomerulonephritis and sepsis, abscesses, osteomyelitis, toxic shock or necrotising fasciitis.

There is reported evidence that the incidence of invasive Streptococcus pyogenes illnesses is increasing [7]. In 2005 the reported incidence of Streptococcus pyogenes invasive infections was 1.5-3.9/100.000 cases per year in developed countries, 6.4-10.2/100.000 in nonindigenous Australian population, 13/100.000 cases at children from Kenya and more than 82/100.00 cases in the indigenous people from Australia [7].

The Gram-positive cocci cause $80 \%$ of the osteoarticular infections. Comparing to Staphylococcus aureus, Streptococcus viridans, and Enterococcus fecalis frequently results in osteomyelitis (56\% respective $57 \%)$ while Streptococcus pyogenes often causes septic arthritis (67\%) [8].

Pyogenic spondylodiscitis with haematogenic pathogenesis firstly affects the lumbar spine (58\%), followed by the thoracic region (30\%) and cervical (11\%) reflecting the vascular characteristics of all these spine levels [8]. The diagnosis is often difficult, and it needs a detailed clinical evaluation, multiple biological and microbiological examinations from a variety of pathological products [9]. The associated symptoms are the chronic fatigue, urinary incontinence occurring as they do in one-third of the patients. $48 \%$ of the patients with spondylodiscitis develop fever [10]. During hospitalization, our patient also associated both symptoms, incontinence, and fever.

A problem related to the present case was the difficulty in confirming the pain source in the absence of changes on the MRI. Haemocultures were performed only when the patient was admitted to the Infectious Diseases service, and it took another seven days before the results were available.

\section{CONCLUSIONS}

This case draws attention to the need to repeat microbiological and imaging examinations for patients with severe illnesses. It is also very important to be constantly aware that the diagnostic reasoning may need to be changed, as the current case demonstrated. In this context, febrile jaundice can be a pitfall but a challenge at the same time.

\section{CONFLICT OF INTEREST}

Nothing to declare

\section{DEFERENCES}

1. Chand N, Sanyal AJ. Sepsis induced Cholestasis. Hepatology. 2007;45:230-41.

2. Singer $M$, Deutschman CS, Seymour CW, et al. The Third International Consensus Definitions for Sepsis and Septic Shock (Sepsis-3). Jama. 2016;315:801-10.

3. Nesseler N, Launey Y, Aninat C, Morel F, Mallédant Y, Seguin P. The liver in sepsis. Critical Care. 2012;16:235.

4. Nguyen KD, Sundaram V, Ayoub WS. Atypical causes of cholestasis. World J Gastroenterol. 2014;20:9418-26.

5. Moseley RH. Sepsis-associated cholestasis. Gastroenterology. 1997;112:302-6.

6. Kosters A, Karpen SJ. The Role of Inflammation in Cholestasis: Clinical and Basic Aspects. Semin Liver Dis. 2010;30:186-94.

7. Ferretti JJ, Stevens DL, Fischetti VA. Streptococcus pyogenes Basic Biology to Clinical Manifestations. Oklahoma City (OK): University of Oklahoma Health Sciences Center. 2016.

8. Murillo O, Grau I, Lora-Tamayo J, et al. The changing epidemiology of bacteraemic osteoarticular infections in the early 21st century. Clin Microbiol Infect. 2015;21:254.

9. Gasbarrini A, Boriani L, Salvadori C, et al. Biopsy for suspected spondylodiscitis Eur Rev Med Pharmacol Sci. 2012;16(Suppl2):26-34.

10. Duarte RM, Vaccaro AR. Spinal infection: state of the art and management algorithm. Eur Spine J. 2013;22:2787-99. 